\title{
An Inner Model Proof of the Strong Partition Property for $\delta_{1}^{2}$
}

\author{
Grigor Sargsyan
}

\begin{abstract}
Assuming $V=L(\mathbb{R})+A D$, using methods from inner model theory, we give a new proof of the strong partition property for $\delta_{1}^{2}$. The result was originally proved by Kechris et al.
\end{abstract}

The main theorem of this note is the following special case of [3, Theorem 1.1] originally due to Kechris, Kleinberg, Moschovakis, and Woodin.

Theorem 0.1 Assume $V=L(\mathbb{R})+\mathrm{AD}$. Then ${\underset{\sim}{1}}_{1}^{2}$ has the strong partition prop-

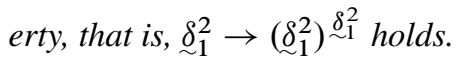

Our proof uses techniques from inner model theory and resembles Martin's proof of strong partition property for $\omega_{1}$ (see Jackson [2]). We expect that it will have other applications and, in particular, can be used to show that under $\mathrm{AD}^{+}$, if $\Gamma$ is any $\Pi_{1}^{1}$-like (i.e., closed under $\forall^{\mathbb{R}}$ and non-self-dual) scaled point class and $\delta=\delta(\Gamma)$, then $\delta$ has the strong partition property. Our motivation to find a new proof of Theorem 0.1 comes from a desire to prove Kechris-Martin-like results for $\Pi_{1}^{1}$-like scaled point classes which will settle Schimmerling [8, Question 19] and most likely, several other questions in the same neighborhood. We are optimistic that inner modeltheoretic techniques will settle this question, and our optimism comes from the fact that the literature is already full of descriptive set-theoretic results that have been proved using methods from inner model theory (see, e.g., Hjorth [1], Sargsyan [5], and Steel [11]). More importantly for us, recently, Neeman, in [4], found a proof of the Kechris-Martin theorem for $\Pi_{3}^{1}$ using techniques from inner model theory. Finally, we believe that our proof can be used to prove the strong partition property for many cardinals $\delta=\delta(\Gamma)$ where $\Gamma$ has strong closure properties. In fact, we expect that it can be used to prove [3, Theorem 1.1], but we certainly have not done so.

We now start proving Theorem 0.1 .

Received May 14, 2012; accepted January 26, 2013

2010 Mathematics Subject Classification: Primary 03E15; Secondary $03 E 45$

Keywords: mouse, inner model theory, descriptive set theory, hod mouse

(C) 2014 by University of Notre Dame 10.1215/00294527-2798745 
Proof of Theorem 0.1 Let $\kappa=\delta_{1}^{2}$. By Martin's theorem (see [2, Theorem 2.31, Definition 2.30]), it is enough to show that $\kappa$ is $\kappa$-reasonable, that is, there is a nonself-dual point class $\underset{\sim}{\Gamma}$ closed under $\exists^{\mathbb{R}}$ and a map $\varphi$ with domain $\mathbb{R}$ satisfying

1. $\forall x(\varphi(x) \subseteq \kappa \times \kappa)$.

2. $\forall F: \kappa \rightarrow \kappa, \exists x \in \mathbb{R}(\varphi(x)=F)$.

3. $\forall \beta<\kappa, \forall \gamma<\kappa, R_{\beta, \gamma} \in \underset{\sim}{\Delta}$ where

$$
x \in R_{\beta, \gamma} \leftrightarrow \varphi(x)(\beta, \gamma) \wedge \forall \gamma^{\prime}<\kappa\left(\varphi(x)\left(\beta, \gamma^{\prime}\right) \rightarrow \gamma^{\prime}=\gamma\right) .
$$

4. Suppose $\beta<\kappa, A \in \exists^{\mathbb{R}} \underset{\sim}{\Delta}$, and $A \subseteq R_{\beta}=\left\{x: \exists \gamma<\kappa R_{\beta, \gamma}(x)\right\}$. Then $\exists \gamma_{0}<\kappa$ such that $\forall x \in A \exists \gamma<\gamma_{0} R_{\beta, \gamma}(x)$.

Let $\Gamma=\Sigma_{1}^{2}$. We claim that $\underset{\sim}{\Gamma}$ is as desired and spend the rest of the proof to argue for it. In what follows, we will freely use the terminology developed for analyzing HOD of models of $\mathrm{AD}^{+}$. This terminology has been exposited in many places including Sargsyan [5], [6], [7], Schindler and Steel [9], Steel [11], and more recently in Steel and Woodin [12]. In particular, recall the definitions of suitable premouse, short tree, maximal tree, and short tree iterable. Given a suitable premouse $\mathcal{P}$, we let $\delta_{\mathcal{P}}$ be its Woodin cardinal and let $\lambda_{\mathcal{P}}$ be the least cardinal which is $<\delta_{\mathcal{P}}$-strong in $\mathcal{P}$.

Suppose $a \in H C$. We say that an $a$-premouse $\mathcal{Q}$ is $\operatorname{good}$ if

1. $\mathcal{Q}$ is $\left(\omega, \omega_{1}\right)$-iterable,

2. $Q \vDash Z$ ZFC - Powerset + "there are no Woodin cardinals" + "there is a largest cardinal,"

3. $\mathcal{Q}$ is full, that is, for every cutpoint $\xi$ of $\mathcal{Q}, L p(\mathcal{Q} \mid \xi) \unlhd \mathcal{Q}$.

If $\mathcal{Q}$ is good, then it has a unique $\left(\omega, \omega_{1}\right)$-iteration strategy with the Dodd-Jensen property. We let $\Sigma_{\mathcal{Q}}$ be this strategy. Also, let $\eta_{\mathcal{Q}}$ be the largest cardinal of $\mathcal{Q}$. Given an iteration tree $\mathcal{T}$ on $\mathcal{Q}$ according to $\Sigma_{\mathcal{Q}}$ with last model $\mathcal{R}$ such that $\pi^{\mathcal{T}}$ exists, we let $\pi_{\mathcal{Q}, \mathcal{R}}: \mathcal{Q} \rightarrow \mathcal{R}$ be the iteration embedding. Notice that because $\Sigma_{\mathcal{Q}}$ has the Dodd-Jensen property, $\pi^{\mathcal{T}}$ is independent of $\mathcal{T}$. We say that $\mathcal{Q}$ is excellent if whenever $\mathcal{R}$ is a $\Sigma_{\mathcal{Q}}$-iterate of $\mathcal{Q}$ such that $\pi_{\mathcal{Q}, \mathcal{R}}$ is defined $\mathcal{R}$ is good. In this case, we also say that $\Sigma_{\mathscr{Q}}$ is fullness preserving.

Suppose now that $\alpha<\kappa$ is such that it ends a weak gap (see Steel [10]). We then let

$$
\mathcal{F}(\alpha, a)=\left\{\mathcal{Q}: J_{\alpha}(\mathbb{R}) \vDash " Q \mathcal{Q} \text { is an excellent } a \text {-premouse" }\right\} .
$$

Given an $a$-premouse $\mathcal{P}$ such that $J_{\alpha}(\mathbb{R}) \vDash$ " $P$ is suitable and short tree iterable," we let $\mathcal{F}(\alpha, a, \mathcal{P})$ be the set of $\mathcal{Q}$ such that in $J_{\alpha}(\mathbb{R})$, there is a correctly guided short tree $\mathcal{T}$ on $\mathcal{P}$ with last suitable model $\mathcal{P}^{*}$ such that for some $\mathcal{P}^{*}$-cardinal $\eta \leq \lambda_{\mathcal{P}^{*}}$, $\mathcal{Q}=\mathcal{P}^{*} \mid\left(\eta^{+}\right)^{\mathcal{P}^{*}}$.

Lemma 0.2 Suppose that $\alpha<\kappa$ ends a weak gap, $a \in H C$, and $\mathcal{P}$ is an a-premouse such that $J_{\alpha}(\mathbb{R}) \vDash " \mathcal{P}$ is suitable and short tree iterable." Then $\mathcal{F}(\alpha, a, \mathcal{P}) \subseteq \mathcal{F}(\alpha, a)$.

Proof Fix $Q \in \mathcal{F}(\alpha, a, \mathcal{P})$. Work in $J_{\alpha}(\mathbb{R})$. Let $\mathcal{T}$ be a correctly guided short tree on $\mathcal{P}$ with last suitable model $\mathcal{P}^{*}$ such that for some $\mathcal{P}^{*}$-cardinal $\eta \leq \lambda_{\mathcal{P}^{*},}, \mathcal{Q}=\mathcal{P}^{*} \mid\left(\eta^{+}\right)^{\mathcal{P}^{*}}$. Because $\mathcal{P}$ is short tree iterable, we have that $\mathcal{Q}$ is $\left(\omega, \omega_{1}\right)$-iterable via a unique iteration strategy $\Sigma$. As the iterations of $\mathcal{Q}$ can also be viewed as iterations of $\mathcal{P}^{*}$, we have that $\Sigma$ is fullness preserving, implying that $\mathcal{Q}$ is excellent. 
Notice that if $\beta>\alpha$ is such that $\beta$ ends a weak gap and $J_{\beta}(\mathbb{R}) \vDash$ " $\mathcal{P}$ is a suitable and short tree iterable $a$-premouse," then there could be $\mathcal{Q} \in \mathcal{F}(\beta, a, \mathcal{P})$ which is not in $\mathcal{F}(\alpha, a, \mathcal{P})$. However, we always have the following easy lemma.

Lemma 0.3 Suppose that $a \in H C, \mathcal{P}$ is an a-premouse and $\alpha<\beta<\kappa$. Suppose that $\alpha$ and $\beta$ end weak gaps such that both $J_{\alpha}(\mathbb{R})$ and $J_{\beta}(\mathbb{R})$ satisfy that $\mathcal{P}$ is suitable and short tree iterable. Then $\mathcal{F}(\alpha, a, \mathcal{P}) \subseteq \mathscr{F}(\beta, a, \mathcal{P})$.

Proof The lemma follows because any iteration tree on $\mathcal{P}$ which is correctly guided and short in the sense of $J_{\alpha}(\mathbb{R})$ is also correctly guided and short in the sense of $J_{\beta}(\mathbb{R})$.

Next we define $\leq_{\alpha, a}$ on $\mathcal{F}(\alpha, a)$ by setting $\mathcal{Q} \leq_{\alpha, a} \mathcal{R}$ if and only if there is an iteration tree $\mathcal{T}$ on $\mathcal{Q}$ according to $\Sigma_{\mathcal{Q}}$ with last model $\delta$ such that $\pi^{\mathcal{T}}$ exists, $\delta \unlhd \mathcal{R}$, and $\delta=\mathcal{R} \mid\left(\eta_{\mathcal{S}}^{+}\right)^{\mathcal{R}}$. Also, let $\leq_{\alpha, a, \mathcal{P}}=\leq_{\alpha, a} \uparrow \mathcal{F}(\alpha, a, \mathcal{P})$. As usual, we have the following.

Lemma 0.4 We have that $\leq_{\alpha, a}$ and $\leq_{\alpha, a, \mathcal{P}}$ are directed, and $\leq_{\alpha, a, \mathcal{P}}$ is dense in $\leq \alpha, a$.

Let then $\mathcal{M}_{\infty}(\alpha, a)$ be the direct limit of $\left(\mathcal{F}(\alpha, a), \leq_{\alpha, a}\right)$ under the iteration embeddings $\pi_{\mathcal{Q}, \mathcal{R}}$. Also, let $\mathcal{M}_{\infty}(\alpha, a, \mathcal{P})$ be the direct limit of $\left(\mathscr{F}(\alpha, a, \mathcal{P}), \leq_{\alpha, a, \mathcal{P}}\right)$ under the iteration embeddings $\pi_{\mathcal{Q}, \mathcal{R}}$. The next lemma follows from Lemma 0.4.

Lemma 0.5 We have $\mathcal{M}_{\infty}(\alpha, a)=\mathcal{M}_{\infty}(\alpha, a, \mathcal{P})$.

We let $\pi_{\mathcal{Q}, \infty}: \mathcal{Q} \rightarrow \mathbb{Q}^{*} \unlhd \mathcal{M}_{\infty}(\alpha, a, \mathcal{P})$ be the direct-limit embedding. ${ }^{1}$

We can now define $\varphi$. First let $S$ be the set of those reals $x$ which code a pair $\left(y_{x}, \mathcal{P}_{x}\right)$ such that

1. $y_{x} \in \mathbb{R}$,

2. for some $\alpha<\kappa$ ending a weak gap, $J_{\alpha}(\mathbb{R}) \vDash$ " $\mathcal{P}_{x}$ is a suitable and short tree iterable $y_{x}$-premouse."

Clearly $S$ is $\Sigma_{1}^{2}$. We let $f: \kappa^{2} \rightarrow \kappa$ be such that $f(\beta, \gamma)$ is the least $\alpha$ such that $J_{\alpha}(\mathbb{R}) \vDash \max (\beta, \gamma)<\delta_{1}^{2}$. We also let $g: S \times \kappa^{2} \rightarrow \kappa$ be the function defined as follows: for all $(\beta, \gamma) \in \kappa^{2}$ and $x \in S$, if there is an ordinal $\alpha>f(\beta, \gamma)$ such that $J_{\alpha}(\mathbb{R}) \vDash$ " $\mathcal{P}_{x}$ is suitable and short tree iterable $y_{x}$-premouse," then $g(\beta, \gamma)$ is the least such $\alpha$, and otherwise $g(x, \beta, \gamma)=0$. Notice that $g$ is $\Sigma_{1}^{2}$ in codes. We define $\varphi$ as follows.

Definition 0.6 If $x \notin S \cap \mathbb{R}$, then let $\varphi(x)=\emptyset$. Suppose now $x \in S$. Let $\left(y_{x}, \mathcal{P}_{x}\right)$ be the pair coded by $x$. Given $\beta, \gamma<\kappa$, we let $(\beta, \gamma) \in \varphi(x)$ if and only if letting $\mathcal{P}=\mathcal{P}_{x}$ and $g(x, \beta, \gamma)=\alpha$, then $\alpha>0$ and for some $a \in \mathcal{P}$ the following holds in $J_{\alpha}(\mathbb{R})$ :

1. $\mathcal{P}$ is suitable and short tree iterable;

2. $a$ is the collapse of $x(0)$;

3. $a \subseteq \lambda_{\mathcal{P}} \times \lambda_{\mathcal{P}}$;

4. there is a correctly guided short tree $\mathcal{T}$ on $\mathcal{P}$ with last model $\mathcal{S}$ such that $\pi_{\mathcal{P}, \mathcal{S}}$ exists and an $\mathcal{\delta}$-cardinal $\eta$ such that

(a) $\left(\eta^{+}\right)^{\mathcal{S}}<\lambda^{\mathcal{s}}$

(b) if $\mathcal{Q}=\& \mid\left(\eta^{+}\right)^{\mathcal{S}}$ and $a^{\mathcal{Q}}=\pi_{\mathcal{P}, s}(a) \cap(\eta \times \eta)$, then $(\beta, \gamma) \in \pi_{\mathcal{Q}, \infty}\left(a^{\mathcal{Q}}\right) \cap$ $\operatorname{rng}\left(\pi_{\mathcal{Q}, \infty}\right)$. 
Given $\alpha<\Theta$, we let $S_{\alpha}, f_{\alpha}, g_{\alpha}$, and $\varphi_{\alpha}$ be what the above definitions give over $J_{\alpha}(\mathbb{R})$. The following lemmas establish that $\varphi$ is as desired. We start with the following easy lemma.

Lemma $0.7 \quad$ For each $x \in \mathbb{R}, \varphi(x)=\bigcup_{\alpha<\kappa} \varphi_{\alpha}(x)$.

Proof Suppose $(\beta, \gamma) \in \varphi(x)$. Let $\alpha>g(x, \beta, \gamma)$ be such that it ends a weak gap. Then $(\beta, \gamma) \in \varphi_{\alpha}(x)$. The other direction is similar.

Lemma $0.8 \quad$ For every $x \in \mathbb{R}, \varphi(x) \subseteq \kappa \times \kappa$.

Proof The claim follows from the fact that for every $\alpha$ and $a, \mathcal{M}_{\infty}(\alpha, a) \subseteq J_{\alpha}(\mathbb{R})$.

Lemma 0.9 Suppose $F: \kappa \rightarrow \kappa$. Then there is $x \in \operatorname{dom}(\varphi)$ such that $\varphi(x)=F$.

Proof Fix $y$ such that $F \in \operatorname{HOD}_{y}$. There is then a suitable $\mathcal{P}$ over $y$ such that $F \in \operatorname{rng}\left(\pi_{\mathcal{P}, \emptyset, \infty}\right) .{ }^{2}$ Notice that $\pi_{\mathcal{P}, \emptyset, \infty}\left(\lambda_{\mathcal{P}}\right)=\kappa$ (see [11, Chapter 8]). Let then $a \subseteq \lambda_{\mathcal{P}} \times \lambda_{\mathcal{P}}$ be such that $\pi_{\mathcal{P}, \emptyset, \infty}(a)=F$, and let $x$ code the pair $(y, \mathcal{P})$ such that $x(0)=a$. It is then easy to see that $\varphi(x)=F$ (use Lemma 0.7$){ }^{3}$

Lemma $0.10 \quad$ Suppose $\beta, \gamma<\kappa$. Let

$$
x \in R_{\beta, \gamma} \leftrightarrow \varphi(x)(\beta, \gamma) \wedge \forall \gamma^{\prime}<\kappa\left(\varphi(x)\left(\beta, \gamma^{\prime}\right) \rightarrow \gamma^{\prime}=\gamma\right) .
$$

Then $R_{\beta, \gamma}$ is $\Delta_{1}^{2}$.

Proof We have that the following are equivalent.

1. We have $x \in R_{\beta, \gamma}$.

2. There is $\alpha$ such that $J_{\alpha}(\mathbb{R}) \vDash " x \in \operatorname{dom}\left(\varphi_{\alpha}\right)$ and $\gamma$ is the unique ordinal such that $(\beta, \gamma) \in \varphi_{\alpha}(x)$."

3. For all $\alpha>f(\beta, \gamma)$ such that $J_{\alpha}(\mathbb{R}) \vDash$ " $x \in \operatorname{dom}\left(\varphi_{\alpha}\right), " \gamma$ is the unique ordinal such that $(\beta, \gamma) \in \varphi_{\alpha}(x)$.

Clearly (1) implies (2) and (3). Also, that (3) implies (1) is straightforward. We show that (2) implies (1). Fix then $\alpha$ such that $J_{\alpha}(\mathbb{R}) \vDash " x \in \operatorname{dom}\left(\varphi_{\alpha}\right)$ and $\gamma$ is the unique ordinal such that $(\beta, \gamma) \in \varphi_{\alpha}(x)$." It follows from the definition of $\varphi_{\alpha}$ that $\alpha>g(x, \beta, \gamma)$. Let $(y, \mathcal{P})$ be the pair coded by $x$, and let $a \in \mathcal{P}$ be the transitive collapse of $x(0)$. Working in $J_{\alpha}(\mathbb{R})$, let $\mathcal{J}$ be a correctly guided short tree on $\mathcal{P}$ with last model $\delta$ such that $\pi_{\mathcal{P}, \mathcal{S}}$ exists and an $\delta$-cardinal $\eta$ such that

1. $\left(\eta^{+}\right)^{8}<\lambda_{s}$;

2. if $\mathcal{Q}=\delta \mid\left(\eta^{+}\right)^{\mathcal{S}}$ and $a^{\mathcal{Q}}=\pi_{\mathcal{P}, \mathcal{S}}(a) \uparrow \eta$, then $(\beta, \gamma) \in \pi_{\mathcal{Q}, \infty}\left(a^{\mathcal{Q}}\right) \cap$ $\operatorname{rng}\left(\pi_{\mathcal{Q}, \infty}\right)$.

Suppose now there is some $\xi$ such that for some $\gamma^{\prime},\left(\beta, \gamma^{\prime}\right) \in \varphi_{\xi}(x)$. Working in $J_{\xi}(\mathbb{R})$, let $\mathcal{T}^{*}$ be a correctly guided short tree on $\mathcal{P}$ with last model $\delta^{*}$ such that $\pi_{\mathcal{P}, \mathcal{S}^{*}}$ exists and an $\boldsymbol{\delta}^{*}$-cardinal $v$ such that

1. $\left(v^{+}\right)^{8^{*}}<\lambda_{8^{*}}$;

2. if $\mathcal{R}=\mathcal{8}^{*} \mid\left(v^{+}\right)^{8^{*}}$ and $a^{\mathcal{R}}=\pi_{\mathcal{P}, \mathcal{S}^{*}}(a) \uparrow v$, then $\left(\beta, \gamma^{\prime}\right) \in \pi_{\mathcal{R}, \infty}\left(a^{\mathcal{R}}\right) \cap$ $\operatorname{rng}\left(\pi_{\mathcal{R}, \infty}\right)$.

Let $v=\max (\xi, \alpha)$. The following is an easy claim.

Claim $\quad J_{v}(\mathbb{R}) \vDash " \&$ and $\varsigma^{*}$ are suitable and short tree iterable." 
Proof We have that $\mathcal{P}$ is suitable and short tree iterable in both $J_{\alpha}(\mathbb{R})$ and $J_{\xi}(\mathbb{R})$. We also have $J_{\alpha}(\mathbb{R}) \vDash$ " $\mathcal{T}$ is short" and $J_{\xi}(\mathbb{R}) \vDash$ " $\mathcal{T}^{*}$ is short." It then follows that $J_{v}(\mathbb{R}) \vDash " \mathcal{T}$ and $\mathcal{T}^{*}$ are short trees on $\mathcal{P}$." It then follows that $J_{v}(\mathbb{R}) \vDash " \varnothing$ and $\delta^{*}$ are suitable and short tree iterable."

We work now in $J_{v}(\mathbb{R})$. Using the claim we can find $8^{* *}$ which is a suitable correct iterate of both $\delta$ and $\delta^{*}$. Notice that since $\delta^{* *}$ is suitable, the iteration embeddings $i: \delta\left|\left(\lambda_{\delta}^{+}\right)^{\delta} \rightarrow \delta^{* *}\right|\left(\lambda_{\delta^{* *}}^{+}\right)^{8^{* *}}$ and $j: \delta^{*}\left|\left(\lambda_{\delta^{*}}^{+}\right)^{8^{*}} \rightarrow \delta^{* *}\right|\left(\lambda_{\delta^{* *}}^{+}\right)^{8^{* *}}$ exist.

Suppose now that $\gamma \neq \gamma^{\prime}$. Let $\left(\bar{\beta}, \bar{\gamma}, \bar{\gamma}^{\prime}\right) \in 8^{* *}$ be such that letting $\zeta=$ $\max \left(i\left(\eta_{\mathcal{Q}}\right), j\left(\eta_{\mathcal{R}}\right)\right)$ and $\mathcal{W}=8^{* *} \mid\left(\zeta^{+}\right)^{8^{* *}}, \pi_{\mathcal{W}, \infty}\left(\bar{\beta}, \bar{\gamma}, \bar{\gamma}^{\prime}\right)=\left(\beta, \gamma, \gamma^{\prime}\right)$. It then follows that $(\bar{\beta}, \bar{\gamma}) \in i\left(\pi_{\mathcal{P}, \mathcal{\mathcal { T }}}^{\mathcal{T}}(a)\right)$ and $\left(\bar{\beta}, \bar{\gamma}^{\prime}\right) \in j\left(\pi_{\mathcal{P}^{*}, \mathcal{S}^{*}}^{\mathcal{T}^{*}}(a)\right)$. However, $i \circ \pi_{\mathcal{P}, \mathcal{S}}^{\mathcal{T}}=j \circ \pi_{\mathcal{P}, \mathcal{S}^{*}}^{\mathcal{T}^{*}}$, implying that $i\left(\pi_{\mathcal{P}, \mathcal{S}}^{\mathcal{T}}(a)\right)=j\left(\pi_{\mathcal{P}^{*} \mathcal{S}^{*}}^{\mathcal{T}^{*}}(a)\right)$ and that

$$
S^{* *} \vDash(\bar{b}, \bar{\gamma}) \in i\left(\pi_{\mathcal{P}, \mathcal{T}}^{\mathcal{T}}(a)\right) \wedge\left(\bar{b}, \bar{\gamma}^{\prime}\right) \in i\left(\pi_{\mathcal{P}, \mathcal{S}}^{\mathcal{T}}(a)\right)
$$

Let now $\left(\tau, \tau^{*}\right) \in \mathbb{Q}$ be such that $\pi_{\mathcal{Q}, \infty}\left(\tau, \tau^{*}\right)=(\beta, \gamma)$. By elementarity of $i$, we then get that $\delta \vDash$ "there is $\tau^{* *} \neq \tau^{*}$ such that $\left(\tau, \tau^{* *}\right) \in \pi_{\mathcal{P}, \delta}(a)$." Fix such a $\tau^{* *}$, and let $\zeta \in\left(\tau^{* *}, \lambda_{s}\right)$ be an $\delta$-cardinal. Then letting $Q^{*}=\delta \mid\left(\varsigma^{+}\right)^{\delta}$ we have that $\left(\beta, \pi_{Q^{*}, \infty}\left(\tau^{* *}\right)\right) \in \varphi_{\alpha}(x)$ and $\pi_{Q^{*}, \infty}\left(\tau^{* *}\right) \neq \gamma$, a contradiction.

The next lemma finishes the proof.

Lemma 0.11 Suppose $\beta<\lambda, A \in \underset{\sim}{\Delta_{1}^{2}}$ and $A \subseteq R_{\beta}=\left\{x: \exists \gamma<\kappa R_{\beta, \gamma}(x)\right\}$. Then $\exists \gamma_{0}<\kappa$ such that $\forall x \in A \exists \gamma<\gamma_{0} R_{\beta, \gamma}(x)$.

Proof Let $h: A \rightarrow \kappa$ be defined by $h(x)=v$ if $v$ is the least such that $v$ ends a weak gap and $J_{v}(\mathbb{R}) \vDash x \in R_{\beta}$. Then $f$ is $\underset{\sim}{\Sigma_{1}}$ over $J_{\kappa}(\mathbb{R})$, and hence, as $\kappa$ is $\mathbb{R}$-admissible, $f$ is bounded.

This completes the proof of Theorem 0.1 .

\section{Notes}

1. Notice that because $\mathcal{Q}$ has a unique iteration strategy, $\pi_{\mathcal{Q}, \infty}$ is independent of $\alpha$ and $a$. Because of this we dropped them from our notation.

2. Recall the direct limit construction that converges to $\operatorname{HOD} \mid \Theta$. Here $\pi_{\mathcal{P}, \emptyset, \infty}$ is the direct limit embedding given by $\emptyset$-iterability embeddings. For more details see either of the aforementioned papers.

3. Notice that by reflection there is $\alpha$ such that $\alpha$ ends a weak gap and $J_{\alpha}(\mathbb{R}) \vDash$ " $\mathcal{P}$ is suitable and short tree iterable." It is then the case that for any $\beta \in(\alpha, \kappa)$ which ends a weak gap, $J_{\beta}(\mathbb{R}) \vDash$ " $\mathcal{P}$ is suitable and short tree iterable."

\section{References}

[1] Hjorth, G., "A boundedness lemma for iterations," Journal of Symbolic Logic, vol. 66 (2001), pp. 1058-72. Zbl 0991.03048. MR 1856727. DOI 10.2307/2695092. 563

[2] Jackson, S., "Structural consequences of AD," pp. 1753-1876 in Handbook of Set Theory, Vols. 1-3, Springer, Dordrecht, Netherlands, 2010. MR 2768700. DOI 10.1007/978-1-4020-5764-9_22. 563, 564 
[3] Kechris, A. S., E. M. Kleinberg, Y. N. Moschovakis, and W. H. Woodin, "The axiom of determinacy, strong partition properties and nonsingular measures," pp. 75-99 in Cabal Seminar 77-79, vol. 839 of Lecture Notes in Mathematics, Springer, Berlin, 1981. MR 0611168. 563

[4] Neeman, I., "An inner models proof of the Kechris-Martin theorem," to appear in The Cabal Seminar, Vol IV, edited by A. S. Kechris, B. Löwe, and J. R. Steel, http://www.math.ucla.edu/ ineeman/ (accessed August 1, 2014). 563

[5] Sargsyan, G., "On the prewellorderings associated with the directed systems of mice," Journal of Symbolic Logic, vol. 78 (2013), pp. 735-63. MR 3135496. 563, 564

[6] Sargsyan, G., "Non-tame mouse from the failure of square at a singular strong limit cardinal," preprint, http://math.rutgers.edu/ gs 481 (accessed August 1, 2012). 564

[7] Sargsyan, G., "A tale of hybrid mice," preprint, http://math.rutgers.edu/ gs481 /hodmice.html. 564

[8] Schimmerling, E., “Open problems," preprint, http://www.math.cmu.edu/users /eschimme/AIM/problems.pdf. 563

[9] Schindler, R., and J. R. Steel, "The core model induction," math.berkeley.edu/ steel. 564

[10] Steel, J. R., "Scales in L(R)," pp. 107-56 in Cabal Seminar 79-81, vol. 1019 of Lecture Notes in Mathematics, Springer, Berlin, 1983. MR 0730592. DOI 10.1007/BFb0071699. 564

[11] Steel, J. R., "An outline of inner model theory," pp. 1595-1684 in Handbook of Set Theory, Vols. 1-3, Springer, Dordrecht, Netherlands, 2010. MR 2768698. DOI 10.1007/978-1-4020-5764-9_20. 563, 564, 566

[12] Steel, J. R., and H. Woodin, "Exploring HOD," to appear in The Cabal Seminar, vol IV, edited by A. S. Kechris, B. Löwe, and J. R. Steel. 564

\section{Acknowledgments}

The author wishes to thank the referees for a list of important corrections. This material is partially based upon work supported by the National Science Foundation under grant DMS-0902628. Part of this paper was written while the author was a Leibniz Fellow at the Mathematisches Forschungsinstitut Oberwolfach.

Department of Mathematics

Rutgers University

Hill Center for the Mathematical Sciences

110 Frelinghuysen Rd.

Piscataway, New Jersey 08854

USA

grigor@math.rutgers.edu 